\title{
« Dirty », le rire et l'angoisse
}

\section{Jean-François Louette}

\section{OpenEdition}

Journals

Édition électronique

URL : http://journals.openedition.org/recherchestravaux/279

DOI : 10.4000/recherchestravaux.279

ISSN : 1969-6434

\section{Éditeur}

UGA Éditions/Université Grenoble Alpes

\section{Édition imprimée}

Date de publication : 30 mai 2005

Pagination : 167-182

ISBN : 0151-1874

ISSN : 0151-1874

\section{Référence électronique}

Jean-François Louette, « «Dirty », le rire et l'angoisse », Recherches \& Travaux [En ligne], 67 | 2005, mis en ligne le 30 septembre 2008, consulté le 08 septembre 2020. URL : http://journals.openedition.org/ recherchestravaux/279; DOI : https://doi.org/10.4000/recherchestravaux.279 
Jean-François LOUETTE

Université Paris 4- Sorbonne

\section{«Dirty», le rire et l'angoisse}

Elle pouffait de rire, un rire idiot, qui n'en finissait plus»... «Et Dirty, dans le vide, éclata de rire sans trouver d'écho ${ }^{\mathrm{I}} » \ldots$ Hérö̈ne du court récit éponyme de Georges Bataille, le personnage devine comme son auteur l'inachevable et la solitude du rire. Ne lit-on pas dans L'Expérience intérieure (1943): "Je riais comme peut-être on n'avait jamais ri, le fin fond de chaque chose s'ouvrait, mis à nu, comme si j'étais mort ${ }^{2}$.» Mon propos ne sera pas de résumer la (difficile) pensée bataillienne du rire ${ }^{3}$; mais plutôt de présenter ce récit - qui a suscité peu d'attention de la part de la critique - où s'entrelacent énigmatiquement le rire et l'angoisse.

On laissera ici les questions de datation que pose «Dirty", et son rattachement à un roman détruit par Bataille, W.-C.4 Bornons-nous à dire, d'une part, que le récit paraît à l'extrême fin de 1945 , mais en portant comme date finale 1928, et que le texte se compose de deux strates inégales. Ce récit daté de 1928 est précédé d'une épigraphe (non datée, mais extraite de la Phénoménologie de l'esprit, qui n'a été traduite en français qu'en 1939 , pour le tome ${ }^{\text {er }}$, et d'une "courte note» - selon le mot de Leiris' - datée, elle, de 1945.

«Dirty» se compose de deux scènes. Dans la première, une femme, Dirty, ivre et belle, montre son sexe dans un bouge, à Londres. La seconde scène se déroule dans une chambre de palace, le Savoy: Dirty évoque devant son ami Léon le souvenir d'une chute grotesque que fit sa mère dans cet hôtel. Ayant

I. Voir "Dirty", dans les Romans et récits de G. Bataille, sous la direction de J.-F. Louette, Paris, Gallimard (Bibliothèque de la Pléiade), 2004, p. 303-309.

2. Euvres complètes, Paris, Gallimard, t. V, I973, p. 46.

3. Voir M. Borch-Jacobsen, «Bataille et le rire de l'être», Critique, janvier I988, p. I6-80.

4. Voir notre notule à «Dirty», Romans et récits, op. cit., p. IIII-III3.

5. Dans sa contribution ("De Bataille l'impossible à l'impossible Documents») au numéro d'hommage de Critique à Bataille, août-septembre 1963, p. 687. Ce texte de Leiris a été repris dans Brisées, Paris, Mercure de France, 1966, puis dans la collection Folio/essais chez Gallimard. 
appelé une femme de chambre et un liftier, elle leur jette un énorme pourboire, puis urine et défeque devant eux. Une fois lavée, elle prend à partie les domestiques, criant sa peur et son écœurement. Enfin elle vomit par la fenêtre, sous le regard de Léon, vide, angoissé.

Miction, défécation, vomissements: voilà qui s'accorde bien avec ce titre du roman de 1925 ou 1926, W.-C. En revanche les dates de 1925 ou 1926 et 1928 ne concordent pas: à sa publication en 1945, dater «Dirty» de 1928, c'est laisser penser que le récit, même s'il appartenait initialement à $W$.-C., a été remanié cette année 1928; mais c'est aussi, semble-t-il, le détacher de W.-C. pour le rapprocher d'Histoire de l'ail, publié précisément en 1928.

\section{Enjeux littéraires}

Sur le plan de l'intratextualité, avant de décrire cette forte parenté avec Histoire de l'œil, il faut signaler les liens entre «Dirty» et les esquisses pour "L'œil pinéal", écrites vers 1927. D’une part, Dirty rappelle cette femme qui se trouve au centre d'un des textes les plus inouïs de Bataille, la description du sacrifice d'un gibbon, mené par "une Anglaise que sa chevelure blonde et auréolée transfigure», et qui « livre son corps admirable à la lubricité et à l'imagination, qu'une étonnante odeur de pourriture achève d'extasier, de plusieurs hommes nus ${ }^{6} »$. D'autre part, Dirty donne à voir un corps qui déborde de luimême - cris, larmes, rires inexplicables, hoquets, rots, puanteur -, et toujours sur le point de se rompre (l'étouffement, le sang au visage): un corps en proie à des "dégagements d'énergie», pour reprendre une formule qui apparaît dans une autre des ébauches sur l'œil pinéal7.

Mais revenons aux traits communs à Histoire de l'œil et à "Dirty». Le dessein d'ensemble est analogue: mettre le lecteur mal à l'aise, voire en état de choc, le suffoquer, selon la courte note ajoutée à «Dirty» en 1945. Le dispositif narratif est le même: un récit au je, conduit aux temps du passé. Le point de vue est masculin, et le récit emploie les verbes sembler ou parâ̂tre pour parler de Dirty ("qui semblait calme», etc.), laquelle demeure énigmatique, tout comme Simone. Le système des personnages est identique: le je masculin se fait le spectateur d'une femme qui se met en jeu sans réserve, allant au bout de l'audace ${ }^{8}$;

6. $O C$, II, p. 28-29.

7. OC, II, p. I9.

8. Voir dans les Romans et récits, p. 893, ces propos de M. d'Ingerville à sa nièce Charlotte: «Elle me disait souvent qu'elle était heureuse d'être une femme, car une femme, surtout belle, surtout riche, pouvait vivre d'une manière plus provocante, à la condition de ne craindre aucune honte, de trouver au contraire sa volupté dans la honte. La volupté était pour elle plus véritable et surtout plus entière que le bonheur qui jamais n'est que la prudence dictée par la crainte de le perdre.» 
Dirty est à l'évidence une sœur de Simone. Léon, lui, est spectateur, mais aussi complice: un couple se voue à l'excès, face à des comparses éteints. Le genre retenu par Bataille, dans les deux cas, n'est pas celui de la nouvelle. Pour cette raison que "Dirty» n'est pas d'abord conçu comme une totalité autonome, mais comme partie de W.-C.; et aussi parce que sur le plan de la composition, il n'y a pas de chute à effet: Bataille remplace même, comme mot de la fin, "où nous pouvions" par "où nous étions".

Sur le plan des thèmes, l'analogie est toujours aussi forte. Laissons le détail des cigares, qui relie le début de "Dirty" à la dernière page d'Histoire de l'œil. Surtout, le motif de la vision est récurrent: du côté de l'ordre, l'« œil éteint» des hommes qui observent Dirty (c'est le «regard châtré» dont parle Histoire de l'œil), et les serviteurs qui se jettent "des coups d'œil obliques"; de l'autre, Dirty qui roule «des yeux ronds et furibonds», a «les yeux hors de la tête»; puis, «le regard mauvais, traqué", "les yeux brûlants de fièvre», elle dit tout «sans rien voir». Chez le narrateur aussi le regard souffre: «mes yeux s'épuisaient». Comme dans Histoire de l'œil, le récit veut conduire vers un invisible.

Affleurent aussi des motifs religieux pervertis (moins nettement que dans Histoire de l'cil, toutefois): la Pieta ("Elle caressa mes tempes»), la chute, l'agenouillement, la souffrance, la plaie ensanglantée, peut-être même l'huile ("une figure marinée dans l'huile»), le sacrifice9, dont Dirty est deux fois la victime ("comme une égorgée», puis "comme un porc sous un couteau»), l'abandon ("comme une petite fille, abandonnée») et l'angoisse, le linge mouillé pour essuyer la figure (on pense au voile de Véronique), l'eau jetée dans la figure (en un contre-baptême), le mot, à la fin du récit, de "créature»... Dirty apparaît comme une sainte de la souillure ${ }^{\text {Io }}$. Son prénom, qui, ainsi qu'il sera expliqué dans Le Bleu du ciel, forme «l'abréviation, provocante, de Dorothea» («don de Dieu»), marque l'ambiguïté du sacré (pur/impur) sur laquelle Bataille insiste à la suite de Hertz, Durkheim ou Frazer; le bouge et les bas-fonds relèvent aussi du sacré impur ${ }^{\mathrm{II}}$.

9. Sur le sacrifice selon Bataille, voir, outre «Le sacrifice du gibbon» dans le "Dossier de l'œil pinéal» (OC, II, p. 28-30), «Le Jésuve» (1930, repris dans $O C$, II, p. I3-I4), et «La notion de dépense» (La Critique sociale, janvier 1933, repris dans OC, I, en particulier p. 306).

Io. Son prénom vaut "de toute évidence, souillure délibérée du prénom "Dorothy" », note Leiris (art. cité, p. 686).

II. Dans le premier numéro de Critique (juin 1946), Bataille écrira: "Un "clochard de Londres" appartient comme un outcast du Bengale au domaine - double - du sacré (éléments les plus purs + tout à fait impurs). Ce qui donne une "horrible grandeur" - inassimilable - à l'homme des bas-fonds de nos villes, n'est nullement étranger au caractère sacré» (repris, légèrement modifié, dans $O C$, XI, p. 6o). 
L'Histoire de l'érotisme, que Bataille écrira en 1950-195I, éclaire rétrospectivement le caractère transgressif du récit. Tout ce qui a marqué le passage de l'animal à l'homme, de «la nature bestiale» à l'humanité est nié par Léon et Dirty. La "pratique du travail»? Mais Léon est «inerte», Dirty jette l'argent par terre, les deux sont "à vau-l'eau». «La nausée de la mort et des morts», qui limite le contact avec les cadavres? Mais c'est sans aucun respect que Dirty évoque sa défunte mère, tombée "les jupes en l'air, un cadavre"; bref, comme dans Histoire de l'œil, la figure maternelle est grotesque. Quant à l'«horreur des besoins animaux" (reproduction, déjections), elle détermine des interdits et des exigences (secret, propreté) que Dirty transgresse en exhibant ses orifices, génésique et excrémentiel - vulve montrée (geste qui annonce celui de Madame Edwarda), main glissée "dans la fente du derrière»-, en vomissant, urinant et déféquant devant témoins. Dirty et Léon évoluent dans le «domaine maudit", celui de l'horreur et de la honte, celui de l'érotisme aussi, vécu dans l'angoisse, car ainsi défini: "découvrir ce qui attire dans le fait qu'un objet est horrible - ou honteux - et faire, devant la nudité honteuse, de la honte et du désir une seule et violente convulsion ${ }^{\mathrm{I2}}{ }$. La convulsion même de Dirty à la première page du récit?

La maigreur du récit rappelle aussi Histoire de l'œil. Mais elle est ici très accentuée; phrases brèves, parataxe prédominante, fréquence des passages à la ligne. Cette sécheresse souligne, par contraste, l'extravagance des conduites racontées. De façon générale, le récit met en œuvre une esthétique du contraste: bouge et palace, robe du soir et immondice, beauté et ignominie, maitres et serviteurs, parfums et puanteur, etc. Les personnages et leurs prénoms se contredisent: Dirty conserve une "candeur intacte»; Léon n'est qu'un lion assez dérisoire.

Au total, «Dirty» forme un récit qui est, comme W.-C., et comme Histoire de l'œil, "assez littérature de fou», pour reprendre l'expression que Bataille emploie dans Le Petit. Une femme et un homme y perdent la tête. D'ailleurs, on devine un arrière-plan freudien: Bataille a emprunté à la Bibliothèque nationale l'Introduction à la psychanalyse (Payot, 1922) de février à juin 1923. On peut remarquer, à la fin du récit, l'emploi du verbe «s'associèrent», ou bien le commentaire de Dirty sur la théière, sans oublier, au début, le symbolisme transparent des cigares éteints. Entre les deux le personnage du liftier fait remonter une scène refoulée du passé de Dirty.

Si l'on quitte l'intratextualité pour l'intertextualité, on songe d'abord à Huysmans: le récit raconte une messe noire ("petites filles jouant au diabolo» [...]), qu'on pourrait comparer à celle de Là-bas: Bataille, qui jeune aimait

I2. $O C$, VIII, p. 67. 
beaucoup Huysmans ${ }^{13}$, emprunte ce roman à la Bibliothèque nationale en 1928, et il évoquera cette messe dans L'Histoire de l'érotisme ${ }^{\mathrm{I}}$.

Mais il faut surtout souligner l'importance capitale de Dostoïevski. On saisit à première lecture ce qui donne au récit son côté Dostoïevski : l'atmosphère de frénésie, l'autohumiliation en public, la prodigalité irraisonnée (on songe à Anastasie Filippovna jetant au feu cent mille roubles dans L'Idiot), le couple de la prostituée et du criminel (sur le modèle de Sonia et Raskolnikov dans Crime et Châtiment), même si Léon ne porte pas encore le nom de ce meurtrier célèbre, Troppmann, qui sera le sien dans Le Bleu du ciel. La première phrase renvoie au Sous-sol, emprunté à la Bibliothèque nationale en juin puis en octobre 1925, texte qui fascinera Bataille, et qui ressortit déjà en un sens à la littérature de fou.

Bataille a lu Dostoïevski à travers l'étude que lui consacre Léon Chestov, qu’il a fréquenté de 1923 à 1925 . Le prénom Léon attribué au protagoniste de "Dirty" signale peut-être cette relation ${ }^{15}$. Cette très belle étude, intitulée "La lutte contre les évidences (Dostoïevski) ", forme la première partie des Révélations de la mort, livre emprunté par Bataille en 1924 - année où il collabore à la traduction d'un autre ouvrage de Chestov, L'Idée de bien chez Tolstoï et Nietzsche ${ }^{16}$. L'étude de Chestov porte avant tout sur Le Sous-sol, intitulé, selon la traduction de son texte par Boris de Schlozer, La Voix souterraine. On ne peut qu'être frappé par l'influence de ces pages sur "Dirty». Le texte de Bataille constitue en un sens une mise en récit de l'analyse de Chestov - mais aussi une mise en excès, conformément à ce que Bataille notera plus tard: «j'eus vite l'impression de différer de lui en raison d'une violence fondamentale qui me portait ${ }^{17}$ », violence d'ordre sexuel, notamment.

Chestov commence par établir une distinction entre deux visions : la "vue naturelle», à laquelle se lie notre raison, et qui passe par des "yeux naturels»; la «seconde vision» octroyée à certains hommes par l'Ange de la Mort: ces "yeux surnaturels» donnent accès à de «nouvelles visions» qui paraissent

I3. Voir, dans une longue note autobiographique du Carnet I4, fo 2 : «Huysmans me semblait dans le monde de la religion le seul qui ait avec mon sentiment quelque chose de commun. J'aimais Huysmans et je m'attachais à tout ce qu'il pensait. » On aura noté la présence de la locution «à vau-l'eau» à la fin du récit.

I4. OC, VIII, p. IIs.

15. Signalons aussi que Bataille signera Léon Noël certains articles de Critique.

I6. Le rapport de Bataille à Chestov a été peu étudié. Voir cependant Michel Surya, «L'arbitraire, après tout. De la "philosophie" de Chestov à la "philosophie" de Georges Bataille», Georges Bataille après tout, D. Hollier éd., Paris, Belin, I995; mais l'article n'aborde pas Les Révélations de la mort.

I7. OC, VIII, p. 563. 
«illégales, ridicules, fantastiques», à la limite de la folie. Cette "seconde vue» fut octroyée à Dostoïevski, et le mena à un certain nombre de découvertes.

D’abord, celle de la réversibilité entre vie et mort: "personne n'est certain que la vie ne soit pas la mort». Dostoïevski aurait compris aussi qu'il n'y a pas de ciel libérateur: «il n'y a de ciel nulle part; il n'y a qu'un «horizon» bas et borné. Les idéals qui élèvent l'âme n'existent pas, il n'y a que des chaînes, invisibles, il est vrai, et qui maintiennent l'homme encore plus solidement que les fers». C'est cette " "vision" nouvelle» qui forme le thème de La Voix souterraine, récit où "l'homme souterrain» est oppressé par "un sentiment torturant du néant", à savoir, "la sensation très nette que cet état d'équilibre, de parfait achèvement, de satisfaction complète considéré par la conscience commune $[\ldots]$ comme l'idéal de la pensée humaine [...] est absolument insupportable». Dans ce récit, Dostoïevski «ne se sent soutenu par aucune autorité, par aucune tradition", car l'expérience humaine "et ses limites, telles qu' elles apparaissent à Kant, et à Comte, ne sont plus aux yeux de Dostoïevski que l'enceinte d'une prison bâtie pour nous par un inconnu ». L'écrivain est attiré par «le "peut-être", l'inattendu, le subit, les ténèbres, le caprice, tout cela justement qui, du point de vue de la science et du bon sens, n'existe pas ou ne doit pas exister». L'homme souterrain, lui, "se dresse», et d'une voix affreuse, «d'une voix qui n'est pas la sienne (la voix de l'homme souterrain, aussi bien que sa vue, ne lui appartient pas en propre)», il défend l'impossible: «Dieu exige l'impossible. Dieu n'exige que l'impossible [...] La métaphysique est impossible! Donc je ne penserai qu’à elle, je ne parlerai plus que d'elle». Une «impulsion intérieure, plus puissante que les intérêts» refuse de considérer l'impossible comme une «muraille de pierre» (formée par le savoir, la science, les lois naturelles). L'homme souterrain consent alors à «l'inertie», il se laisse écraser, humilier, pour échapper à la contrée où règnent les lois, celle où vivent les gens normaux, modérés, aristotéliciens, qui suscitent "dans la région souterraine un rire homérique». L'homme souterrain ose cesser de croire au bon droit de la raison. Il clame qu'il est "parfois très agréable de briser quelqu'un». Chestov lui donne, par prosopopée, la parole: "Je ne défends pas ici la souffrance ou le bien-être, mais je suis pour mon caprice $[. .$.$] je suis sûr que l'homme ne renoncera jamais à la vraie souffrance,$ c'est-à-dire à la destruction et au chaos. » Qu'est-ce que le caprice? "L'homme souterrain ne possède pas de but clair, défini. Il veut ardemment, passionnément, follement, mais il ne sait pas ce qu'il veut et ne le saura jamais. » Aussi, «il ne pense pas; il s'agite, désespéré; il frappe de tous côtés, il cogne sa tête à tous les murs. Il s'enflamme sans cesse, il atteint les cimes les plus élevées pour se précipiter ensuite dans Dieu sait quels abîmes». C'est d'ailleurs un trait commun à tous les héros de Dostö̈evski : «ses héros ne savent pas agir, ils ne 
savent pas créer, ils ne le veulent même pas, semble-t-il; la destruction, la mort les suivent pas à pas, afin, probablement, de ne pas donner au lecteur l'illusion même d'une conclusion ". Et Chestov d'ajouter: "Tous les héros de Dostoïevski aspirent à ce qui menace de les perdre.» Lui-même «renonce à la certitude et pose comme but suprême - l'ignorance».

On reconnaît dans ces pages un grand nombre des thèmes sur lesquels Bataille construit «Dirty»: le rejet du bon sens, de la mesure, des lois, de l'idéalisme, de la raison, l'accès, dans une insatisfaction essentielle, à une autre vision, fascinée par la mort, l'indéfini, l'ignorance (le non-savoir), la perte de soi, le caprice, l'impossible - et le rire. Chestov reviendra sur son analyse de «l'homme souterrain» dans La Philosophie de la tragédie. Dostoüewski et Nietzsche ${ }^{\mathrm{I} 8}$; par là il entend ces êtres qui ont renoncé à la sensiblerie, à l'humanitarisme, à l'idéalisme, non sans une souffrance tragique. Mettant en jeu avec Dirty et Léon deux de ces êtres souterrains, donc blessés - à la main, pour Léon - et tragiques, mais se rappelant aussi que toute tragédie se fonde sur un sacrifice, Bataille, prenant Chestov au mot, raconte un sacrifice humain, dont Dirty est la victime volontaire.

Cependant, la grande différence qui sépare Bataille de Dostoïevski (et encore plus nettement de Chestov), est l'importance que le premier accorde à une figure féminine scandaleuse. La dissymétrie du couple ne peut manquer de frapper: orgasme et audace de Dirty, passivité et impuissance de Léon. Cette dissymétrie fait songer à celle qu'évoquera Bataille en mars 1953, dans un article intitulé «Hemingway à la lumière de Hegel», à propos du roman The sun also rises, paru, indique Bataille de mémoire, en 1925 (en fait en I926, et la traduction française, par Maurice-Edgar Coindreau, à la NRF, en 1933). Il est possible que Bataille, sur les conseils de Queneau, l'ait lu entre 1926 et 1928. En tout cas ce qu'il en dit éclaire «Dirty». Car le couple de Dirty et Léon reproduit en un sens celui de la fascinante Brett Ashley («il n’est pas de personnage dans le livre qui ait le prestige de Brett", "en sa personne, l'ivresse est souverainement séduisante») et de Jake Barnes, ivrogne, lui, parce

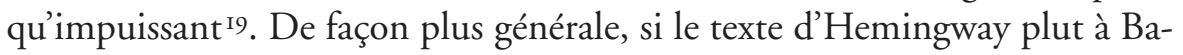
taille, comme à toute une génération, c'est que, dit-il, contre la tradition de la mesure française "nous aimions la fièvre et la sauvagerie, tout ce qui est immédiatement et directement». Bataille fut aussi très sensible chez Hemingway à ce qu'il résume en une formule qui définit assez bien «Dirty " : la «quête

I8. Traduction B. de Schlœzer, I926; c'est l'un des ouvrages de Chestov auxquels Bataille fait allusion («Léon Chestov philosophait à partir de Dostoïevski et de Nietzsche, ce qui me séduisait", OC, VIII, p. 563).

19. Pour ce rapprochement, voir B. Mattheus, "Koinzidenzen», Das Blau des Himmels, Matthes \& Seitz, I990, p. 189-190. 
de la souverainetée ${ }^{20}$. En ce sens, il est logique que Dirty compare sa mère à une reine, et le prénom Léon (lion...) n’est pas immotivé.

La date de 1928 constitue aussi le récit en réponse à Nadja. (Ou : Bataille le relit après coup comme une réponse à $\mathrm{Nadja}$ ). Car 1928 est l'année où Breton publie Nadja (fin mai), avec sa célèbre dernière phrase: «La beauté sera CONVULSIVE ou ne sera pas». Bataille, qui trouve Breton prude et timoré en matière de sexualité ${ }^{21}$, trop proche des communistes orthodoxes en matière de politique, enchaîne: la femme, osons la dépoétiser, la convulsion sexuelle, osons la représenter. Osons même lui attribuer une force révolutionnaire?

Apparaissent alors les trois différences capitales qui séparent "Dirty" d'Histoire de l'œil. D'une part, point de rattachement autobiographique: certes Bataille est passé par Londres en 1920, où il raconte avoir dîné avec Bergson $^{22}$, certes le récit pourrait faire fonds sur des "souvenirs d'enfance», mais une seule phrase, à la dernière page du récit, oriente en ce sens, et sans rien préciser. D’autre part, Bataille opère un déplacement vers un terrain politique, puisque la scène se place à Londres, à la fois haut lieu du capitalisme (l'hôtel Savoy) et ville fortement liée au marxisme (l'édition originale du Manifeste du Parti communiste y fut imprimée en I848, Karl Marx y mourut). Or - et troisième différence avec Histoire de l'ail - ce terrain est aussi philosophique, comme le soulignera le titre d' «Introduction » donné à "Dirty» au moment de son remaniement et de son insertion dans Le Bleu du ciel en 1957.

\section{Enjeux politico-philosophiques}

Bataille est grand lecteur de Nietzsche depuis 1923. Le récit présente une allure nietzschéenne, en raison de la séparation nette entre la morale terne des esclaves, et l'immoralité supérieure des maîtres. Mais avec deux inflexions proprement batailliennes. Du côté des maîtres, le sentiment du vide, l'abaissement recherché. D'autre part, ce qui vient sur le devant de la scène, et qui d'ailleurs ouvre le texte, au prix d'une distorsion de l'ordre des événements, c'est l'orgasme suggéré de Dirty.

Bataille a toujours donné pour décisive, dans sa formation intellectuelle, sa lecture de l' "Essai sur le don", paru dans l'Année sociologique de 1923-1924, publiée effectivement en 1925. C'est seulement fin mai I93I qu'il emprunte cette revue à la Bibliothèque nationale. Mais Alfred Métraux lui avait parlé de

20. OC, XII, p. 246 et 255 .

2I. Voir la réponse de Breton à l'Enquête sur l'amour dans le dernier numéro de La Révolution surréaliste, Is décembre 1929 (reprise dans Euvres complètes, t. I, Paris, Gallimard (Bibliothèque de la Pléiade), p. IO2O-IO22), et l'éloge de la monogamie dans L’Amour fou.

22. $O C$, VIII, p. 562 . 
Mauss, dont il suivait les cours, et de son enseignement sur le potlatch, dès I922, ou $1925^{23}$. Mauss a permis à Bataille de placer à l'origine de l'économie le potlatch, la dépense somptuaire agonistique, qui produit la lutte des classes en ce qu'elle oppose les nobles qui dilapident et les ignobles par eux voués à l'infériorité et à la déchéance 24 . Dirty réaliserait l'idéal du potlatch selon Mauss: elle va au bout de la dépense improductive, physique (l'exhibition, les sécrétions, le rire) et financière (l'énorme pourboire), sans que ce don soit rendu. Car si dans son analyse théorique de 1933, Bataille soutient que le contre-don (le moment où la dépense sociale est reprise par le prolétariat) est constitué par la Révolution, dans le récit les domestiques ne donnent à Dirty en échange de ses excès que leur peine respectueuse.

On peut donc lire «Dirty» comme une scène de politique expérimentale. Le motif solaire dans le récit (les "lumières crues", les «cheveux blonds, sous les lumières", "la lumière nue $[. .$.$] aveuglante») et dans la courte note («[...]$ plutôt qu'un dieu, être un soleil») reconduit à L'Anus solaire. Or on y trouve la proposition suivante: "ceux en qui s'accumule la force d'éruption sont nécessairement situés en bas 25 ». Un des enjeux du récit n'est-il pas de rompre avec cette idée? En bas, du côté du liftier et de la femme de chambre, nulle force éruptive. Le liftier ne fait pas monter le peuple dans l'ascenseur révolutionnaire. Il n'y a d'éruption que chez Dirty (au prix, il est vrai, de son abaissement) : elle seule est le "canon" qui décharge.

Le thème de la lutte des classes n'apparaissait guère dans Histoire de l'œeil. Mais dans "Dirty", on a bien, face à deux bourgeois, deux "domestiques", deux travailleurs. Surtout, du liftier, il est dit d'emblée qu'il «avait l'air d'un fossoyeur»: faut-il voir là une allusion à une phrase fameuse de Marx et Engels dans le Manifeste du Parti communiste, selon laquelle ce que la bourgeoisie "produit avant tout, ce sont ses propres fossoyeurs ${ }^{26}$ "? Bataille emprunta cet opuscule à la Bibliothèque nationale en juillet 1933, mais on peut penser qu'il avait déjà lu ce texte célèbre, puisqu'il le citait dans son article de mars 1932 écrit avec Queneau, «La critique des fondements de la dialectique hégélienne 27 ». Or le prolétariat du Savoy ne réagit pas aux provocations de Dirty: il conserve obstinément sa "parfaite déférence». Le fossoyeur révolutionnaire de Marx devient un liftier, rivé à sa «cage» (le mot est

23. En 1922 selon A. Métraux, «Rencontre avec les ethnologues», Critique, août-septembre 1963; Bataille, lui, se rappelle - en I949 - que c'est «aux environs de 1925» que Métraux lui parla avec enthousiasme de l'Essai sur le don (OC, VII, p. 484).

24. Voir "La notion de dépense», La Critique sociale, janvier I933, OC, I, p. 3 Iо.

25. $O C$, I, p. 85 .

26. Fin du chap. $\mathrm{I}^{\mathrm{er}}$.

27. OC, I, p. 289. 
dans le texte), et la «servante» se voue au rangement, à l'ordre. Ils demeurent asservis au travail. Où est donc passée la combativité du prolétariat, moteur de la lutte des classes? Le prénom de Léon pourrait alors renvoyer ironiquement à Trotski, grand défenseur de la dictature prolétarienne, dont Bataille emprunte plusieurs livres en $1926^{28}$. Ironie, parce que la prétendue révolution permanente chère à celui que Bataille nommera (en 1953) «le plus brillant des théoriciens communistes 29 » depuis la mort de Lénine en 1924, aboutirait en fait à une inertie - à laquelle s'opposerait la force de l'explosion sexuelle qu'incarnerait Dirty? Ou bien: la révolution permanente ne retrouverait de force qu'à s'associer à l'énergie sexuelle? Bataille, quand il reprendra en 1935 son texte pour l'insérer dans Le Bleu du ciel, se demandera plus nettement s'il n'est pas possible de capter cette énergie sexuelle à des fins révolutionnaires.

Bataille note plus d'une fois que la dialectique du maitre et de l'esclave est «à l'origine de la théorie communiste de la lutte des classes» (ainsi dans son article sur Genet, repris dans La Littérature et le Mal). Derrière Marx, il conviendrait donc de chercher Hegel. En 1945, l'épigraphe cite, plus longuement que ne le faisait Kojève quand il avait isolé, traduit et commenté les mêmes lignes en $1934^{30}$, un passage de l'introduction de la Phénoménologie, dans la traduction d'Hyppolite (quelque peu modifiée). Voilà qui invite à rapprocher "Dirty» des analyses hégéliennes.

Cette épigraphe correspond à une stratégie complexe de la part de Bataille ${ }^{31}$. D'un côté, elle indique, conformément à l'usage, les significations globales du récit: marquer l'impossibilité, vécue dans l'angoisse, de se contenter d'une "satisfaction limitée», manifester, à travers les personnages de Dirty et Léon, le désir de dépasser toutes les bornes, dans une mort de l'être naturel donné qui seule est "humanisante ou anthropogène ", pour reprendre les mots que Bataille avait pu entendre de Kojève ${ }^{32}$. L'épigraphe hégélienne s'accorde alors non seulement avec l'enseignement de Chestov lisant Dostö̈evski, mais

28. Signalons que B. Souvarine, lui-même grand défenseur de Trotski jusqu'en I929, dans le "Prologue" à la réédition de La Critique sociale en 1983 (Paris, Éditions de la Différence, p. 2I), s'offusque du fait que Bataille, pour le dénigrer (sans qu'on sache exactement dans quel contexte), ait emprunté le prénom de son frère (Léon) tué à la guerre le 4 avril I9I5 (comme le précise J.-L. Panné, Boris Souvarine, Paris, Robert Laffont, I993, p. 37). Souvarine viset-il «Dirty»? Chestov, Trotski ou Léon Souvarine: ce prénom fait décidément signe vers la Russie.

29. "La souveraineté», OC, VIII, p. 308.

30. Voir son Introduction à la lecture de Hegel, Paris, Gallimard (rééd. Tel), 1947, 1979, p. 552553.

3I. Elle relève de ce que J.-M. Besnier nomme «une répétition différentielle» de Hegel chez Bataille (La Politique de l'impossible. L'intellectuel entre révolte et engagement, Paris, La Découverte, 1988, p. 152).

32. Introduction à la lecture de Hegel, op. cit., p. 553. 
aussi avec tel passage de L'Expérience intérieure, où Bataille, en 1943, fait de l'angoisse et de l'inconnu un moment sur le chemin de l'extase - «Souvent l'inconnu nous donne de l'angoisse, mais il est la condition de l'extase»-, et enfin avec l'importance capitale de la mort dans la pensée de Bataille ${ }^{33}$. Signe de cet accord avec Hegel, c'est très exactement le même verbe - suffoquer qui vient sous la plume de Bataille pour exprimer l'impression que fit sur lui le cours de Kojève consacré à la Phénoménologie 34, et, selon la note liminaire, l'effet que doit produire "Dirty». Se relisant en 1945 , Bataille ne put qu'être frappé par la consonance de son récit avec certains thèmes hégéliens: aussi décide-t-il d'emprunter une épigraphe à la Phénoménologie.

"Dirty» serait ainsi un récit hégélien? Non, et l'épigraphe, d'un autre côté, fonctionne comme un leurre. Bataille publie sa fiction contre l'auteur même à qui il emprunte une épigraphe. D'une part, sous le patronage de Hegel se place un texte qui en fait met en scène tout ce dont Hegel ne rend pas compte. Le 5 février 1938, dans un exposé au Collège de sociologie, Bataille notera que la "Phénoménologie de l'Esprit, écrite en I806, s'est passée des données de Freud et de Mauss ${ }^{35}$ "; on ne saurait donc se borner à la répéter ou à l'interpréter. D'où ces précisions, cinq ans plus tard: "Mes efforts, écrit Bataille dans L'Expérience intérieure, recommencent et défont la Phénoménologie de Hegel»; celle-ci est "une philosophie du travail, du "projet" ", elle néglige "l'existence non discursive, le rire, l'extase», ou encore «l'ivresse sacrée", et on pourrait ajouter: les larmes, la fièvre, les déchirures ${ }^{36}$. La Phénoménologie, comme Bataille le précisera en 1955, a tort de trop accorder "au sérieux du malheur et à l'absence de jeu37 »; à quoi s'oppose, dans "Dirty», l'insistance sur le rire. Ce qui est en question, c'est enfin la validité d'un mode de pensée non abstrait: on pense, dans le monde de Bataille, aussi par le rire, les larmes, etc., comme déjà chez Nietzsche et chez Dostoïevski $3^{8}$.

D'autre part, en vertu de la force de l'ambivalence chez Bataille, même si le récit insiste sur le rire, il recentre aussi l'attention sur ce que Hegel finit par négliger, à savoir l'angoisse; l'on comprend alors pourquoi l'épigraphe est empruntée à l'introduction de la Phénoménologie: Bataille veut ne retenir que

33. OC, V, p. 169 .

34. «Explication géniale, à la mesure du livre. Combien de fois Queneau et moi sortîmes suffoqués de la petite salle - suffoqués, cloués.» (OC, VI, p. 416)

35. OC, II, p. 322.

36. Voir L'Expérience intérieure, OC, V, p. 96.

37. "L'au-delà du sérieux", La Nouvelle NRF, n² 26, février 1955, OC, XII, p. 315.

38. C'est ce que Bataille avait pu lire dans La Philosophie de la tragédie: "chez Nietzsche, penser signifier souffrir, se torturer, se rouler dans des convulsions. / Les héros de Dostoïewski ne pensent pas non plus conformément aux règles de la logique: ce ne sont chez lui que sanglots, grincements de dents, efforts frénétiques» (op. cit., p. 198). 
ce début du mouvement hégélien, où l'angoisse a encore sa place, mais il rejette l'aboutissement du processus dialectique - pour le dire vite, l'accomplissement de la conscience de soi, la confusion parfaite du réel et du rationnel, la fin de l'Histoire, qui lui semble impliquer une liquidation de l'angoisse 39.

Enfin, on peut penser que le récit conteste la dialectique hégélienne du maître et de l'esclave. Bataille la connaissait-il déjà quand il écrit "Dirty»? Il n'en est pas question dans la brève Histoire de la philosophie allemande d'Émile Bréhier (I92I), qu'il emprunte à la Bibliothèque nationale de juillet à novembre 1924. Cependant, cette dialectique du combat pour la reconnaissance est exposée par Hegel non seulement dans la Phénoménologie (I807), mais aussi, dix ans plus tard, dans la troisième partie de l'Encyclopédie, la Philosophie de l'Esprit: or Bataille, fin 1925 début 1926, emprunte, durant un mois et demi, la traduction de cet ouvrage par A. Véra ${ }^{40}$. On peut donc penser qu'il s'est alors au moins formé une idée de cette fameuse analyse. Il en fait d'ailleurs mention dans son article de mars 1932 sur «La critique des fondements de la dialectique hégélienne ${ }^{4 \mathrm{I}}$. Certes, il datera de 1933 sa "véritable entrée en contact avec l'œuvre de Hegel $4^{2} »$ : mais n'est-ce pas manière de rendre hommage à Kojève, dont le cours, devenu célèbre, professé à l'École des Hautes Études, débute en 1933 ? Ce cours portait plus précisément sur la Phénoménologie, dont Bataille n'empruntera une édition en allemand, à la Bibliothèque nationale, qu'en décembre $1934^{43}$.

Dans la version qu'en donne la Philosophie de l'esprit, les moments clés du processus sont les suivants: l'une des consciences en conflit l'emporte parce qu'elle méprise sa «naturalité», son «être naturel»; cependant «le serviteur, en travaillant pour le maître, use sa volonté individuelle et égoïste, supprime l'immédiatité [sic] du désir, et par cette abdication de lui-même, et par la crainte du maître amène le commencement de la sagesse 44 ». Or dans "Dirty", les maitres, d'une part, n'échappent pas plus que les esclaves à la naturalité, si

39. Dans son Histoire de la philosophie allemande, que Bataille a lue en I924, Bréhier écrit ceci: "Hegel parle quelque part de l'“angoisse" de l'esprit qui se sent isolé dans un monde d'objets étrangers et extérieurs à lui, où il ne se reconnaît pas. Cette angoisse, ce sentiment d'étrangeté des choses, exprimées par bien d'autres métaphysiciens allemands [...] sont bien, semble-t-il, les ressorts cachés de la pensée de Hegel; toute sa philosophie est une philosophie qui veut nous rassurer et supprimer cette angoisse.» (Paris, Payot, I92I, p. I2O)

40. OC, XII, p. 562 . L'analyse de la lutte pour la reconnaissance se lit, dans la Philosophie de l'esprit, aux $\$ 43 \mathrm{I}-436$ de la trad. de A. Véra (t. II, p. 64-80), aux $\$ 430-435$ (p. 230-232) de la trad. procurée par B. Bourgeois (Paris, Vrin, 1988).

4I. $O C, \mathrm{I}, \mathrm{p} .278$.

42. Notice autobiographique de 1958, OC, VII, p. 615.

43. OC, XII, p. 598.

44. Traduction de A. Véra, Philosophie de l'Esprit, Paris, Germer Baillère, t. II, I869, p. 69, 70, 77. Cette sagesse est la «conscience de soi générale» (Véra), « universelle» (Bourgeois). 
l'on en juge par la répartition équitable des métaphores animalisantes: le liftier se voit comparé à un "gorille», Dirty à un "porc», sa mère à un "dogue». D'autre part, il se peut que le liftier et celle que Dirty nomme «l'honnête fille» disposent d'une sagesse - mais, suggère Bataille, au prix de quelle platitude, face à l'éclat de Dirty! En fait, alors que Hegel (et Kojève y insistera en 1934), défend à l'évidence la valeur positive du travail, c'est pour Bataille le travail luimême qu'il convient de rejeter 45 .

Docile à la suggestion de l'épigraphe, confrontons maintenant "Dirty" à la version de la même dialectique que donne non plus l'Encyclopédie mais la Phénoménologie - et tout d'abord à l'analyse que Bataille entendit Kojève en faire, et qui avait été publiée en $1939^{46}$. Le contraste entre le récit et la dialectique hégélienne - Bataille a dû le sentir en se relisant en 1945 - est alors encore plus fort, pour deux raisons. Pour devenir le maitre, Kojève y insiste, il faut surmonter l'angoisse (ce mot est absent des paragraphes que traduit Véra) - mais chez Bataille les maîtres eux-mêmes n'ont pas l'air d'y parvenir («moi aussi j’ai peur, mon garçon», etc.); d'autre part, il est nécessaire de dépasser «la nature animale empirique 47 »: au rebours on a vu que les métaphores y renvoient les personnages du récit. Kojève aurait décidément dit mais a-t-il jamais lu «Dirty»? - que le processus "anthropogène» ne s'y accomplit point de façon conforme à ce que décrit Hegel 48 .

Compliquons encore un peu les choses. Dans l'épigraphe, Bataille cite (modifiée, toutefois) la traduction de la Phénoménologie par Hyppolite en I939. Lecteurs de cette traduction, c'est alors à un « renversement » de la servitude en "véritable indépendance 49 » que nous nous attendons - lequel n'advient certes pas dans «Dirty».

Concluons sur ce qu'engage ici le débat avec Hegel. D’une part, la fiction fonctionne à la fois comme illustration d'analyses avec lesquelles Bataille se sent en accord (Nietzsche, Mauss), comme réfutation de théories constituées (Marx), et comme laboratoire de pensée (le rapport à Hegel) : bref, comme un

45. Introduction à la lecture de Hegel, op. cit., p. 56. Bataille: entre tant d'énoncés qui vont dans le même sens, il écrira en I953, dans «La souveraineté» : «le travail est l'exact contraire de l'attitude souveraine» (OC, VIII, p. 324).

46. «Le passage sur le maître et l'esclave de la Phénoménologie de l'Esprit [...] est traduit et commenté par A. Kojève dans le numéro de Mesures du I5 janvier 1939" (L’Expérience intérieure, $O C, \mathrm{~V}, \mathrm{p} . \mathrm{I} 28)$.

47. Introduction à la lecture de Hegel, op. cit., p. 52 et 57I. Répétons-le: dans le texte que traduit Véra, il n'est question que de la «naturalité de la vie» (op. cit., p. 75).

48. "Anthropogène» est un mot récurrent sous la plume de Kojève, voir par ex. Introduction à la lecture de Hegel, op. cit. p. 57I-572.

49. G.W.F. Hegel, La Phénoménologie de l'esprit, trad. J. Hyppolite, Paris, Aubier-Montaigne, I94I, t. I, p. I63. 
lieu de mise à l'épreuve du spéculatif. D'autre part, le jeu des dates (1945/1928) entre lesquelles Bataille encadre son récit nous permet, dociles à l'épigraphe, de faire intervenir dans notre lecture de "Dirty» un texte de Hegel, la Phénoménologie, que Bataille n'avait très probablement pas encore lu en 1928, mais dont, pour ce qui est de sa partie la plus célèbre (le maître et l'esclave), il avait découvert une autre version, dans la Philosophie de l'esprit, en 1925. Enfin, l'épigraphe mêle peut-être à dessein deux traductions (Kojève/Hyppolite), afin que l'on ne sache plus au juste à laquelle confronter «Dirty»... Comme si cette épigraphe qui parle de l'angoisse mettait aussi en place un vertige une angoisse - de lecture.

\section{Publier en 1945}

Revenons à la fin de l'année 1945. Pourquoi Bataille tire-t-il «Dirty» de l'ombre à ce moment-là, pour le publier sous son nom? C'est la première fois qu'il avoue ainsi un de ses récits érotiques. Qu'est-ce qui rendrait "Dirty" moins scandaleux qu'Histoire de l'œil? L'absence, on l'a dit, de rattachement autobiographique? Et aussi l'absence de scènes de coïts? Une chose est sûre: la comparaison du texte publié avec le dactylogramme du texte de 1928 conservé à la Bibliothèque nationale de France montre que Bataille au dernier moment a atténué les audaces de son récit: ainsi, à la première page, «Elle étirait les jambes et se branlait» devient "Elle étirait les jambes et s'amusait", "exhibant l'immondice de la fente " cède la place à "montrant l'immonde de la fente», et le mot "orgie» est supprimé. Plus loin, "Ma mère s'est cassé la gueule» devient «Ma mère s'est cassé la figure », et «Elle a dégueulé» est évincé au profit de «Elle a vomi». Bataille remplace "L'urine forma une flaque» par "Une flaque se forma", il substitue à "couilles» des points de suspension, réduit "masturbez» à son initiale, etc.

On peut aussi se demander quel rôle a joué la publication de L'Expérience intérieure au début de 1943 . En un sens, elle a donné à Bataille un autre statut, d'écrivain «important», qui n'a plus besoin, dans "Dirty», d'afficher certains signes convenus de la littéraritéso, et qui peut se permettre de renoncer au pseudonyme? Ou bien L'Expérience intérieure ayant défait la notion même d'auteur, et en tout cas d'autorité de l'auteur, Bataille se sent plus libre de publier, sous son nom mais sans pour autant assumer une responsabilité entière, un récit scandaleux, pourvu qu'il soit sans clair fondement autobiographique (à la différence d'Histoire de l'ail lors de sa réédition clandestine de 1947, ou du Petit en 1943)?

50. Bataille renonce ainsi, en 1945, aux adjectifs antéposés («ignoble bouge», «intolérable éclat», "intacte candeur»). 
Quant au fait même que Bataille ait décidé de publier "Dirty», il s'explique d'abord par des raisons circonstancielles, d'ordre financier, Bataille souffrant chroniquement du manque d'argent. Dans le carnet 8 (daté de I945), $\mathrm{f}^{\circ}$ 52, dans une liste de rentrées d'argent attendues, on trouve: «Parisot Io 000 ». Or Henri Parisot dirigeait aux Éditions Fontaine la collection dans laquelle parut «Dirty».

Il est possible aussi qu'ait joué un rôle un regain d'intérêt, chez Bataille, pour Le Sous-sol: le I9 juin 1946 la radio française en diffusera, sous le titre L'Esprit souterrain, une adaptation par Bataille et sa cousine germaine MarieLouise $^{\varsigma \mathrm{I}}$; Bataille la fait donc précéder par ce récit ancien qui s'inspire fortement du Sous-sol.

Par ailleurs, Bataille éprouve le besoin de préciser sa position dans le champ des idées philosophiques. En raison de l'importance qu'il accorde à l'angoisse, on le rattache souvent alors à Heidegger, et il s'en agace à l'extrême: "Je ne veux pas être mis à la suite de Heidegger", écrit-il dans un carnet daté de 1945-1946, ajoutant qu'il considère Sein und Zeit comme un «travail professoral ${ }^{52}$ ", qui ne permet de penser ni la souveraineté et la révolte qu'elle implique, ni l'érotisme, et qui néglige le rire - si fréquent en revanche chez Dirty, et fondamental dans la pensée de Bataille. Par l'épigraphe il s'agit donc d'indiquer que le véritable débat se situe non point avec Heidegger, mais avec Hegel. Toutefois, pour marquer ses distances avec l'auteur de la Phénoménologie, il lui oppose à la fois l'existence non discursive et un mode d'expression non discursif: une fiction.

De plus, en publiant un récit qui peut en 1945 se lire comme une contestation de la valeur que la dialectique hégélienne accorde au travail, comme une mise en cause de l'ardeur du prolétariat dans la lutte des classes, comme une défense de la dépense inconditionnée, Bataille entend peut-être suggérer (non sans provocation en un temps de pénurie) que l'après-guerre devrait renoncer à l'utilité, au travail, à l'accumulation: le récit formerait alors, par anticipation, un équivalent plus accessible et plus frappant de La Part maudite, que Bataille en 1945 pense achever sous peu, mais qu'il ne publiera qu'en 1949 chez Minuit.

Enfin, la publication non clandestine de «Dirty» correspondrait au désir de prendre position dans le débat d'actualité sur l'articulation de la littérature et de la politique (le premier numéro des Temps modernes, avec la «Présentation" de Sartre, paraît le I ${ }^{\text {er }}$ octobre 1945$)$. Dans le cadre du projet qu'il nourrit de

5I. Cette adaptation a été retrouvée dans les archives de Radio-France par C. Moscovitz, qui l'a publiée, avec une présentation, dans L'Infini, $\mathrm{n}^{\circ} 75$, été $200 \mathrm{I}$.

52. Carnet 9, $\mathrm{f}^{\circ}$ II (cité dans $O C, \mathrm{~V}, \mathrm{p} .474$ ) et f ${ }^{\circ} 92$. 
consacrer le deuxième cahier de la revue Actualité à «Littérature et politique», Bataille écrit à Brice Parain, le 29 juin 1945: «Le langage littéraire est comparable aux objets de luxe, retirés du circuit de la production pour une dépense improductive. [...] la littérature exprime seule ce mouvement de fête 53 ». Le récit "Dirty» est une fête : à la fois par son contenu (l'orgie, la dépense improductive sous toutes ses formes) et par le fait même de son appartenance à la littérature.

Un objet retiré du circuit: il faut, pour finir, remarquer comment la «courte note» de 1945, qui s'ouvre par un regret («Dommage!») et évoque une meilleure version possible de l'histoire, ressortit à la rhétorique du désœuvrement (contestation de l'œuvre dans l'œuvre même) qui apparaît dans tous les récits diurnes de Bataille, «relevant» la stratégie des pseudonymes, au sens hégélien du verbe (remplacer en conservant, dépasser en subsumant). Le texte prend dès son début soin de suggérer sa propre annulation: longtemps soustrait au public, il ne lui est donné, que pour continuer, d'une autre manière, à se dérober.

De même s'est ici dérobée l'analyse du rire. Non seulement parce que, selon un paradoxe constant dans les textes narratifs de Bataille, même si Dirty rit, «Dirty» ne fait guère rire. Mais aussi parce que face au rire, la théorie est "courte». Tel fut le sentiment de Bataille après la lecture du Rire de Bergson en 192054. Deux commentaires précisent l'immense portée de cette déception. L'un se lit dans un fragment autobiographique qui date peut-être de I958: «le problème du rire me parut sans discussion le fondement. Je n'imaginais pas que rire me dispensât de penser, mais que rire, étant à certains égards préalable à ma pensée, me porterait plus loin que la pensée55». L'autre se trouve dans L'Expérience intérieure: "la question, le sens demeuré caché du rire, fut dès lors à mes yeux la question clé [...], l'énigme qu’à tout prix je résoudrai (qui, résolue, d'elle-même résoudrait tout) ${ }^{56}$ ». Mais l'énigme du rire peut-elle se résoudre? Et doit-on en conclure que le dernier mot - du récit, de la pensée de Bataille - revient à l'angoisse?

53. G. Bataille, Choix de lettres, M. Surya éd., Paris, Gallimard, I997, p. 239-240.

54. L'Expérience intérieure, $O C, \mathrm{~V}, \mathrm{p} .80$.

55. OC, VIII, p. 562.

56. $O C, \mathrm{~V}, \mathrm{p} .80$. 